\title{
Cochlear Implantation for Single-Sided Deafness: Observations in Poor Performers
}

\author{
Philipp Mittmann $^{1} \quad$ A. Ernst ${ }^{1} \quad$ S. Scholz ${ }^{1} \quad$ R. D. Battmer ${ }^{1} \quad$ I. Todt ${ }^{1}$
}

1Department of Otolaryngology, Head and Neck Surgery, Unfallkrankenhaus Berlin, Germany

Ann Otol Neurotol ISO 2018;1:18-22

\begin{abstract}
Address for correspondence Philipp Mittmann, MD, Department of Otolaryngology, Head and Neck Surgery, Unfallkrankenhaus Berlin, Warener Str. 7, 12683 Berlin, Germany (e-mail: philipp.mittmann@ googlemail.com).
\end{abstract}

Abstract

Keywords

- single-sided deafness

- cochlear implant

- sound localization
Objectives Patients with single-sided deafness can nowadays receive a cochlear implant. A majority of these patients are well adapted and benefit from the implant. The aim of this study was to evaluate the reasons for poor performance in a group of patients with single-sided deafness, who received a cochlear implant.

Design A total of 65 patients were enrolled into a retrospective case series. Seven poor performers were present in the group. Freiburger monosyllabics, localization testing, and radiologic images were evaluated.

Results Localization testing showed the absence of lateralization ability in three patients, whereas the Freiburger monosyllabic word scores improved in three patients. One patient had no speech perception after 1 year of rehabilitation. Findings of magnetic resonance imaging (MRI) revealed cerebral involvement in five patients.

Conclusion Various factors influence the outcome in unilateral cochlear implantation in patients with single-sided deafness. These reasons may be preoperative, operative, or postoperative. Nevertheless, the majority of patients benefit from these implants.

\section{Introduction}

Cochlear implantation (CI) is a globally accepted surgical procedure for an increasing number of patients with severe to profound hearing loss. The criteria for $\mathrm{CI}$ have expanded over the past decades. Single-sided deafness (SSD) is characterized by severe to profound sensorineural hearing loss in one ear and normal hearing in the contralateral ear. $\mathrm{CI}$ is a well-established option for hearing rehabilitation in patients with SSD. ${ }^{1}$ Nowadays, patients with SSD make up a nonnegligible percentage of all $\mathrm{CI}$ recipients in countries with cost coverage for this group.

Beside CI treatment, the options for SSD include conventional hearing aids, conventional contralateral routing of sound (CROS) hearing aids, and bone-anchored hearing aids (BAHAs). The latter offer patients pseudo-binaural hearing but cannot restore a profoundly deaf ear. Compared with pseudo-binaural hearing solutions (BAHA or CROS), CIs restore binaural hearing and can improve hearing abilities significantly. ${ }^{1}$ As with adults, children with SSD have objectively and subjectively binaural and bilateral hearing benefits from $\mathrm{Cl}^{2}{ }^{2}$

Difficult hearing situations, such as hearing in noise or hearing at greater distances, are facilitated by binaural hearing. ${ }^{3}$ Improvements in hearing abilities with a $\mathrm{Cl}$ are especially beneficial for open set speech recognition in noise and for sound localization. ${ }^{4}$

Sound localization is a fundamental auditory skill. Localization abilities are based on interaural time differences (ITDs) and interaural level differences (ILDs), both of which require the use of binaural hearing, which people with SSD do not have. However, to make full advantage of ITD cues, artificial electrical stimulation by the $\mathrm{CI}$ needs to be translated into neural activity.

Nevertheless, before CI, several candidacy considerations have to be obtained. Friedmann et al point out absolute indications (late-stage unilateral Menière's disease, an "at risk" only hearing ear, and pediatric progressive hearing loss) for 
CI. ${ }^{5}$ Several other factors, such as age and duration of hearing loss, are well known and have to be considered, as the benefits from a CI might not be satisfactory.

Since these factors influence the outcome overtime, based on patient experiences with bilateral hearing loss and CIs, it is of special interest to look at the group of patients with SSD. It can be assumed that, based on the asymmetric hearing situation, different factors than those in the previous group influence the outcome.

The aim of this study was to investigate a group of patients who presented themselves after unilateral CI for SSD with nonsatisfactory hearing ability in our department.

\section{Materials and Methods}

The study was reviewed and supported by the institutional review board (IRB-ukb-HNO-2016/04). Preoperatively, computed tomography (CT) scan of the temporal bone and magnetic resonance imaging (MRI) of the neurocranium were performed. Cases with any malformation were excluded. In cases of long-term hearing loss (> 20 years), promontorial testing was performed. $\mathrm{CI}$ included a mastoidectomy and a posterior tympanotomy as well as a round window or modified round window approach to access the cochlea. The senior author performed all surgeries. Out of a series of 65 patients with SSD, seven patients with subjective poor performance were included in this study. All patients met the criteria for unilateral CI in an SSD situation with normal hearing in the contralateral ear. Audiologic testing included speech perception testing in a sound field using the German Freiburger test for monosyllabic words at $65 \mathrm{~dB}$ sound pressure level (SPL) with a signal coming from the front. The Freiburger test for monosyllabic words was measured at the initial fitting and after 3, 6, and 12 months. All patients underwent audiologic rehabilitation after the initial fitting. In some patients, localization testing was performed in a room with 47 loudspeakers in a semicircle with a 2.35-m distance to the patient; all speakers were covered. Two different stimuli with 750-milliseconds noise bursts were applied. Low-frequency bursts were between 300 and 1,200 $\mathrm{Hz}$ and high-frequency bursts between 2,000 and $8,000 \mathrm{~Hz}$ at $65 \mathrm{~dB}$. Sound was presented from a determined angle. The patient had to point in the exact direction of the sound origin.

\section{Results}

Seven patients (three females and four males) were included in this study. Mean age at implantation was $60.82 \pm 16.75$ years with a mean duration of hearing loss of $6.98 \pm 11.25$ years. Six of seven patients had a speech recognition test available up to 1 year after implantation. The Freiburger monosyllabic word score (FWS) showed mean values of $14.29 \pm 18.58$ at the initial fitting, $23.57 \pm 19.30$ at 3 months, $24.17 \pm 24.58$ at 6 months, and $31.67 \pm 31.09$ at 12 months. Analysis of variance (ANOVA) for repeated measures showed no significant difference in FWS over time with $p=0.285$. Three patients completed the lateralization testing.
1. Patient 1 underwent $\mathrm{CI}$ surgery 2 years after idiopathic sudden hearing loss. He presented with subjective poor performance in the right ear after CI. FWS was $45 \%$ at the initial fitting and dropped to $25 \%$ at the 3 -month visit. Over the first year, the patient improved to $55 \%$ at 6 months and $75 \%$ at 12 months. Retrospective analysis of cerebral MRI before implantation revealed several microembolisms in the area of the right middle cerebral artery. Sound localization testing at high and low frequencies showed for the right (CI)-side lateralization to the left side at $10,30,60$, and 90 degrees. In summary, lateralization ability did not improve with the $\mathrm{Cl}$ ( - Fig. 1A, B).

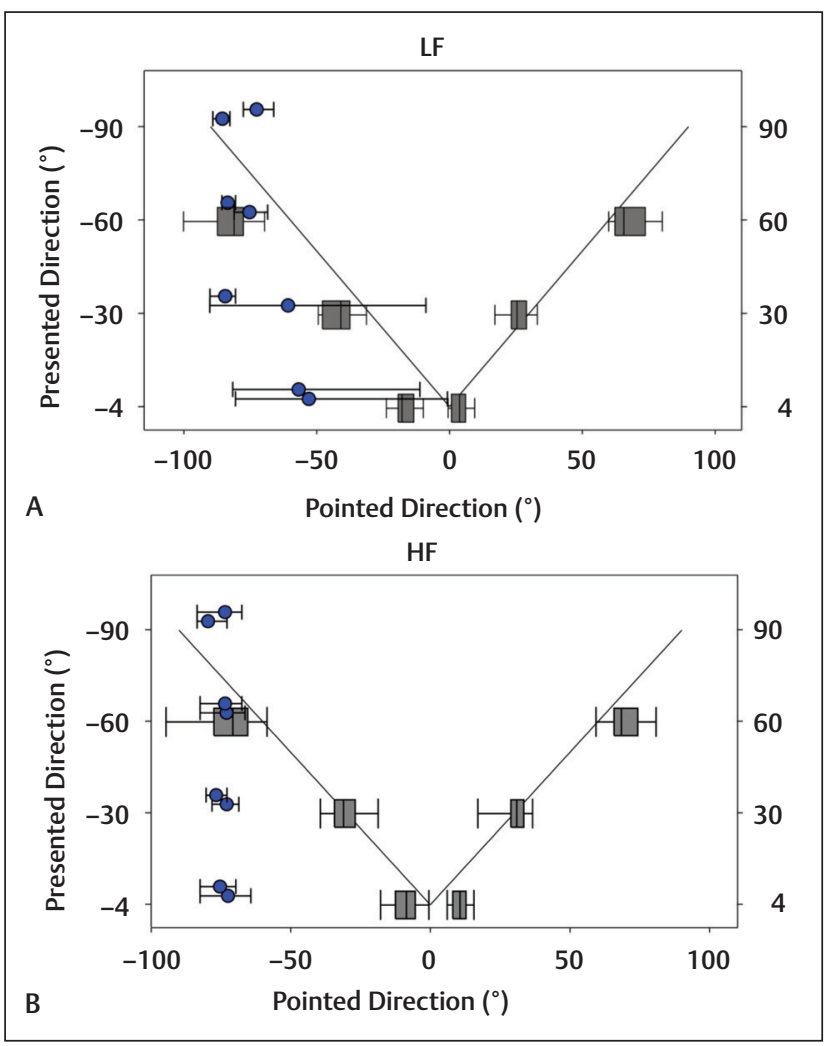

Fig. 1 Localization testing in the (A) low (LF) and (B) high frequencies (HF) in patient 1. The patient has a cochlear implant on the right side.

2. Patient 2 had a progressive hearing loss on the right side after multiple middle ear surgeries due to a cholesteatoma. He underwent implantation of a vibrant sound bridge, which improved the hearing ability temporarily. After 7 years of hearing loss, he underwent CI. FWS improved from $0 \%$ at the initial fitting to $15 \%$ at 3 months, $30 \%$ at 6 months, and $35 \%$ at 12 months. Because of an unsatisfactory hearing outcome, the patient wore the audio processor only occasionally, even during auditory rehabilitation.

3. Patient 3 underwent $\mathrm{CI}$ on the left side 18 months after sudden hearing loss due to an assumed rupture of the round window membrane. Preoperative CT and MRI scans were inconspicuous. Postoperative radiologic examination revealed that the electrode array translocated from the scala tympani into the scala vestibuli. FWS increased from $0 \%$ at the initial fitting to $35 \%$ at 3 months but decreased to 
20\% after 12 months. During auditory rehabilitation, the patient claimed adaption problems (voices sounded like hammer blows) and started slowly to retreat socially.

4. Patient 4 was referred to our department with bacterial meningitis due to acute mastoiditis on the right side. Four days after mastoidectomy, a placeholder was implanted as the patient reported substantial hearing loss on the right side evidenced by pure-tone audiometry (PTA). Two months after meningitis, a CI was implanted without any complications. After the initial fitting and during the year of rehabilitation, the patient did not report any hearing sensation. An MRI revealed cerebral residuals after meningitis with cerebral microangiopathia, which led to an impairment of the central auditory pathway and an additional cerebral ischemic insult ( - Fig. 2A, B).
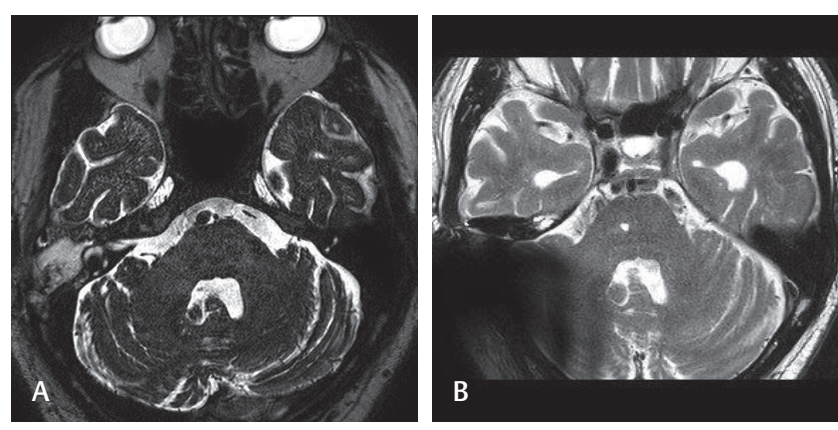

Fig. 2 MRI scan before (A) and after (B) cochlear implantation on the right side. T2-weighted images after cochlear implantation include the artifact caused by the magnet. The lacunar infarct is seen in the pontine area.

5. Patient 5 had 4 years of unilateral hearing loss following a sudden hearing loss before $\mathrm{CI}$. The preoperative cerebral magnet response imaging (cMRI) showed a lacunar infarct on the right side of the frontal lobe. FWS increased from $35 \%$ at the initial fitting to $60 \%$ at 3 months and remained rather steady until 12 months. Sound localization testing in low frequencies on the right $(\mathrm{CI})$ side showed a deviation to the left side at 5 degrees. At 30 degrees, localization testing on the right side was out of the normal distribution. In the high-frequency range, at all tested frequencies, sound provided to the right ear was lateralized to the left side, indicating the inability to localize with the $\mathrm{CI}(-$ Fig. 3A, B).

6. Patient 6 had a history of unilateral hearing loss for 2 years after a car accident, which resulted in a temporal bone fracture and cerebral bleeding, resulting in a lesion in the left pontine area. She reported poor performance and showed an FWS score of $15 \%$ at the initial fitting and at 3 months, which decreased to $10 \%$ at 6 months and $0 \%$ at 12 months. Localization testing in the low- and high-frequency ranges showed abrogated localization ability in both the ears; most presented sounds were localized in the portion between 50 and -50 degrees ( - Fig. 4A, B).

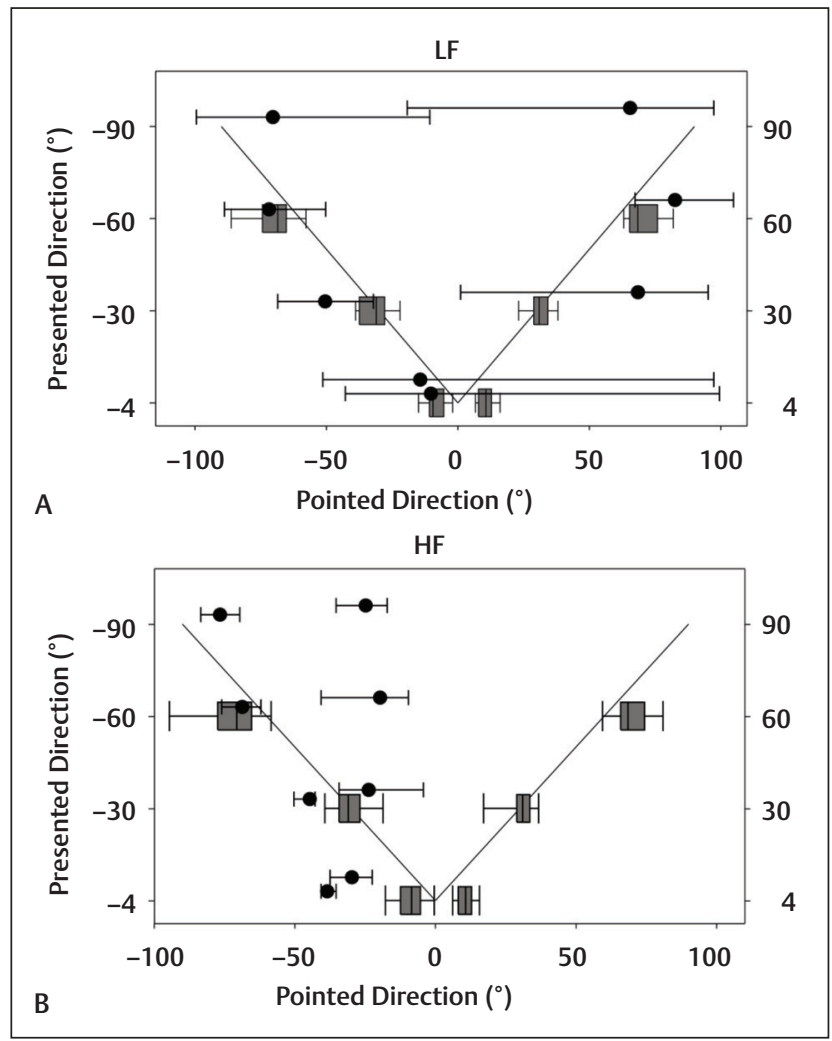

Fig. 3 Localization testing in the (A) low (LF) and (B) high frequencies (HF) in patient 5 . The patient has a cochlear implant on the right side.

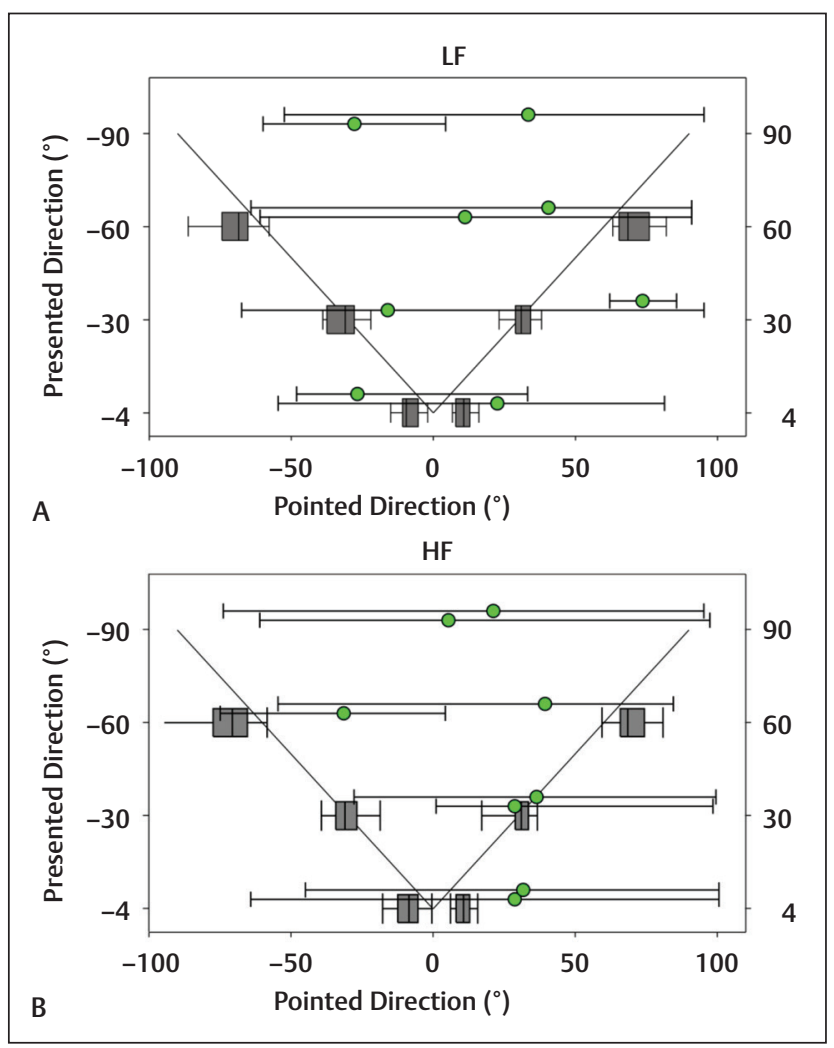

Fig. 4 Localization testing in the (A) low (LF) and (B) high frequencies (HF) in patient 6 . The patient has a cochlear implant on the right side. 
7. Patient 7 was implanted after progressive hearing loss over 15 years. She reported low speech perception at the initial fitting and only wore the audio processor 2 hours a day. FWS scores were $15 \%$ at the initial fitting and $5 \%$ at 3 months. The preoperative MRI showed severe subcortical white matter lesions in both the hemispheres and linear signal accentuations periventricularly.

\section{Discussion}

Cochlear implantation for patients with severe to profound hearing loss has become a standard surgical procedure in recent years. However, the criteria and patient populations have changed. Hearing abilities can certainly be improved by a CI, especially for open set speech recognition in noise and for sound localization. ${ }^{1,4}$ In contrast to the pseudo-binaural benefits of different treatment options, adults with SSD significantly benefitted from a CI. ${ }^{2}$ Zhang et al showed that the bilateral integration of acoustic and electric auditory stimulation via the normal hearing ear and the contralateral $\mathrm{CI}$ improved performance in speech perception. ${ }^{6}$

We present a small case series of nonsatisfying outcomes and poor performance after $\mathrm{CI}$ implantation in patients with unilateral deafness. The etiologies of the poor $\mathrm{Cl}$ performance were variable and could be separated into three types: preoperative, operative, and postoperative.

Preoperative reasons included patients 1, 5, 6, and 7. Patient 1 showed several microembolisms preoperatively on MRI scans in the area of the right middle cerebral artery. Although scoring satisfactorily on the FWS test, the sound localization test revealed a missing ability to localize sound with the normal hearing ear and the CI. Cerebral microembolisms can directly influence central auditory pathway transmission and may influence the CI outcome. Patients 5, 6, and 7 showed intracerebral lesions as well. Patient 5 had a lacunar infarct area on the right side of the frontal lobe and patient 6 had a lesion in the left pontine area. The pons contains the cochlear nuclei ascending fibers, which are a part of the auditory pathway. Disturbance of these fibers can result in defective transmission, which may lead to limited speech perception and limited sound localization. Lesions in the frontal lobe should not influence speech perception and sound localization, but it could not be completely excluded that further lesions (although not seen on MRI) impaired the auditory pathway. Patient 7 had white matter lesions in the subcortical frontoparietal part of the brain and early-stage degenerative cerebral microangiopathy. These focal lesions may impair brain connectivity, which has recently been termed "connectional diaschisis" and reflects changes that occur to brain regions distant from the location of the lesion. ${ }^{7}$ While connections to and from lesioned areas are likely to suffer owing to lost afferents, ${ }^{8}$ connectional diaschisis can also involve connectivity changes between distal regions that are not directly connected to the lesion site. Therefore, the outcome remains unclear and is not easily predictable. As the $\mathrm{Cl}$ has more advantages than CROS or bone conduction devices in patients with unilateral hearing loss, ${ }^{1}$ the indication for the $\mathrm{Cl}$ should be favorable in those cases with potential structural damage.
Operative reasons included patient 3. This patient showed a scalar dislocation of the electrode array on postoperative radiologic images. Electrode extrusion into the scala vestibuli causes disappointing audiologic outcomes as word scores correlate negatively with the number of electrodes in the scala vestibuli., ${ }^{910}$ Speech recognition results, up to 1 year after implantation, were poor in this patient. As preoperative MRI scans revealed no cerebral pathology and the duration of deafness was short, the scalar rearrangement of the electrode array can be regarded as crucial.

Postoperative reasons for poor performance were found with patient 4. Bacterial meningitis can result in cochlear obstruction secondary to labyrinthitis ossificans. ${ }^{11}$ Labyrinthitis ossificans can impede $\mathrm{Cl}$ and result in incomplete insertion. ${ }^{12}$ Patient 4 suffered from bacterial meningitis due to acute mastoiditis. He received a placeholder and underwent $\mathrm{Cl}$ with full electrode insertion and no sign of labyrinthitis ossificans after 3 months. Nevertheless, the patient did not have any speech perception due to lesions in the central auditory pathway and stopped using the audio processor after 1 year. Further diagnostics included a cMRI, which revealed cerebral residuals after meningitis with cerebral microangiopathy and a recent postoperative cerebral ischemic insult.

Various reasons were found to impair the functional outcome after $\mathrm{CI}$ in this study group. To provide a sufficient and high-quality $\mathrm{CI}$ program, all factors surrounding implantation should be assessed. These especially include pre- and postoperative radiologic evaluations as well as audiologic tests. The magnet within the $\mathrm{Cl}$ induces an artifact. Minimization of the artifact can be achieved by careful placement of the device at the right angle and distance to the external auditory canal and by selecting specific technical scanning sequences on MRI scans. ${ }^{13}$

This study has some limitations. Despite the anatomic and pathophysiologic conditions, psychologic and psychophysical reasons may influence outcomes, benefits, and quality of life with a CI. ${ }^{14}$ This study addresses only anatomic and pathophysiologic conditions.

The majority of $\mathrm{CI}$ recipients benefit from the implant. As the indication for $\mathrm{CI}$ has been extended, patients with SSD have become a certain part of the patient collective. The benefits for patients with SSD include sound localization and speech comprehension in the majority of subjects. ${ }^{15}$ The authors present here a small selected group of individuals who did not benefit from a Cl in terms of speech recognition and/or sound localization. For the first time, the authors have shown in single cases, presumably related to specific defects in the auditory pathway, that although improved speech perception can be achieved, localization abilities do not develop after $\mathrm{Cl}$ surgery in SSD.

\section{Conclusion}

Cochlear implantation for the treatment of patients with SSD has become a standard procedure. Despite some cases with poor performance after $\mathrm{CI}$, the majority of patients perform well with the implant. Nevertheless, careful selection of patients and pre- and postoperative radiologic evaluations are needed. 


\section{Funding}

No funding was available for this study.

\section{Ethical Approval}

All procedures performed in studies involving human participants were in accordance with the ethical standards of the institutional and/or national research committee and with the 1964 Helsinki declaration and its later amendments or comparable ethical standards.

\section{Informed Consent}

Informed consent was obtained from all individual participants included in the study.

\section{Conflict of Interest}

None.

\section{References}

1 Arndt S, Aschendorff A, Laszig R, et al. Comparison of pseudobinaural hearing to real binaural hearing rehabilitation after cochlear implantation in patients with unilateral deafness and tinnitus. Otol Neurotol 2011;32(1):39-47

2 Arndt S, Prosse S, Laszig R, Wesarg T, Aschendorff A, Hassepass F. Cochlear implantation in children with single-sided deafness: does aetiology and duration of deafness matter? Audiol Neurootol 2015;20(Suppl 1):21-30

3 Brown KD, Balkany TJ. Benefits of bilateral cochlear implantation: a review. Curr Opin Otolaryngol Head Neck Surg 2007; 15(5):315-318

4 Vermeire K, Van de Heyning P. Binaural hearing after cochlear implantation in subjects with unilateral sensorineural deafness and tinnitus. Audiol Neurootol 2009;14(3):163-171
5 Friedmann DR, Ahmed OH, McMenomey SO, Shapiro WH, Waltzman SB, Roland JT Jr. Single-sided deafness cochlear implantation: candidacy, evaluation, and outcomes in children and adults. Otol Neurotol 2016;37(2):e154-e160

6 Zhang T, Spahr AJ, Dorman MF. Frequency overlap between electric and acoustic stimulation and speech-perception benefit in patients with combined electric and acoustic stimulation. Ear Hear 2010;31(2):195-201

7 Carrera E, Tononi G. Diaschisis: past, present, future. Brain 2014;137(Pt 9):2408-2422

8 Campo P, Garrido MI, Moran RJ, et al. Remote effects of hippocampal sclerosis on effective connectivity during working memory encoding: a case of connectional diaschisis? Cereb Cortex 2012;22(6):1225-1236

9 Lassig AA, Zwolan TA, Telian SA. Cochlear implant failures and revision. Otol Neurotol 2005;26(4):624-634

10 Skinner MW, Holden TA, Whiting BR, et al. In vivo estimates of the position of advanced bionics electrode arrays in the human cochlea. Ann Otol Rhinol Laryngol Suppl 2007;197:2-24

11 Isaacson B, Booth T, Kutz JW Jr, Lee KH, Roland PS. Labyrinthitis ossificans: how accurate is MRI in predicting cochlear obstruction? Otolaryngol Head Neck Surg 2009;140(5):692-696

12 Liu CC, Sweeney M, Booth TN, et al. The impact of postmeningitic labyrinthitis ossificans on speech performance after pediatric cochlear implantation. Otol Neurotol 2015;36(10):1633-1637

13 Todt I, Rademacher G, Mittmann P, Wagner J, Mutze S, Ernst A. MRI Artifacts and cochlear implant positioning at $3 \mathrm{~T}$ in vivo. Otol Neurotol 2015;36(6):972-976

14 Olze H, Szczepek AJ, Haupt H, Zirke N, Graebel S, Mazurek B. The impact of cochlear implantation on tinnitus, stress and quality of life in postlingually deafened patients. Audiol Neurootol 2012;17(1):2-11

15 Blasco MA, Redleaf MI. Cochlear implantation in unilateral sudden deafness improves tinnitus and speech comprehension: meta-analysis and systematic review. Otol Neurotol 2014;35(8):1426-1432 